Удк 676.1+681.121.4

\title{
ДОСТОВЕРНОСТЬ МОНИТОРИНГА ОБОРОТНЫХ ВОД ЦЕЛЛЮЛОЗНО-БУМАЖНОГО ПРОИЗВОДСТВА \\ С ПОМОЩЬЮ ПОГРУЖНЫХ ВИХРЕВЫХ РАСХОДОМЕРОВ
}

\author{
(C) Ю.Д. Алашкевич, М.С. Лурье, О.М. Лурье, А.С. Фролов*
}

Сибирский государственный аэрокосмический университет

им. М.Ф. Решетнева, пр. Мира, 82, Красноярск, 660049 (Россия)

В работе освещается вопрос эксплуатации погружных вихревых расходомеров, предназначенных для мониторинга оборотных вод целлюлозно-бумажного производства.

Рассмотрены различные составляющие (геометрическая и гидродинамическая) общей погрешности измерения данных расходомеров. Подробно рассмотрены погрешности монтажа приборов как самых ответственных операций, предшествующих их вводу в эксплуатацию. На графиках представлены результаты расчетов геометрической составляющей погрешности, вызванные неперпендикулярностью характеристического размера тела обтекания (ТО) и оси трубопровода, а также от смещения осей ТО и сечения трубопровода. Показано, что изменение геометрии проточной части погружного расходомера приводит и к изменению процесса вихреобразования, в результате чего возникает дополнительная погрешность, которая является гидродинамической составляющей общей погрешности. Для нахождения гидродинамической составляющей погрешности был использован метод численного моделирования гидродинамических процессов. Представлены зависимости погрешности монтажа на гидродинамическую составляющую общей погрешности. Выявлено, что на величину погрешности оказывает влияние не только поворот и смещение ТО, но и типоразмер трубопровода, что в свою очередь связано с процессом вихреобразования.

Ключевые слова: оборотные воды, тело обтекания, вихревые расходомеры, погрешности монтажа, имитационное моделирование.

\section{Введение}

Экологические и экономические проблемы, связанные с производственной деятельностью предприятий целлюлозно-бумажной промышленности (ЦБП), невозможно решить без осуществления мониторинга сточных и оборотных вод, одной из составляющих которого является строгий учет их объема.

Для измерения количества сточных и оборотных вод необходимо соответствующее расходоизмерительное оборудование: расходомеры и водосчетчики.

Как известно, потребление свежей воды и, соответственно, количество стоков в ЦБП во много раз превосходит объем выпуска продукции. Поскольку все производства данной отрасли многотоннажные, то расходы оборотных вод (в зависимости от стадии производства) могут измеряться десятками и тысячами тонн воды в час [1].

Алашкевич Юрий Давыдович - заведующий кафедрой машин и аппаратов промышленных технологий, доктор технических наук, професcop, e-mail: mapt@sibgtu.ru Лурье Михаил Семенович - профессор кафедры автоматизации производственных процессов, доктор технических наук, e-mail: m_o_1@rambler.ru Лурье Ольга Михайловна - доцент кафедры автоматизации производственных процессов, кандидат технических наук, e-mail: m_o_1@rambler.ru

Фролов Александр Сергеевич - доцент кафедры автоматизации производственных процессов, кандидат технических наук, e-mail: frolov-a84@mail.ru
Поэтому важной метрологической особенностью расходомеров и водосчетчиков для ЦБП является возможность измерения расхода в трубопроводах больших диаметров и открытых каналах.

Поскольку стоки ЦБП несут в себе большое количество взвешенных частиц и химических веществ, то приборы для измерения их расхода должны быть работоспособны в загрязненных и агрессивных жидкостях [2].

\footnotetext{
* Автор, с которым следует вести переписку.
} 
Погружные вихревые расходомеры являются новыми перспективными приборами, предназначенными для измерения расхода различных технологических жидкостей ЦБП и, в частности, применимыми к учету оборотных вод [3].

Погружные расходомеры устанавливаются непосредственно в рабочий трубопровод, что упрощает конструкцию измерительного преобразователя и трудоемкость его монтажа.

Общая погрешность измерения таких приборов будет складываться из:

- погрешности изготовления и монтажа приборов;

- погрешности, вызванной шероховатостью внутренней поверхности трубопровода, которая влияет на профиль скоростей потока [4];

- температурных погрешностей;

- погрешности метода измерения - $\Delta S h, \%$, как отклонение от среднего значения числа Струхаля $(S h)$ в диапазоне изменения скоростей движения жидкости в трубопроводе (рис. 1). Эта погрешность зависит от формы тела обтекания (ТО) расходомера, некоторые виды которых рассмотрены в работе [5].

Рассмотрим подробнее погрешности монтажа приборов в трубопроводе с целью выработки норм допусков на установку таких расходомеров.

Монтаж расходомера на рабочем трубопроводе является одной из самых ответственных операций, предшествующих его вводу в эксплуатацию. При погружной конструкции вихревых расходомеров возникает ряд погрешностей, связанных с неточностью или ошибками при данной операции [6]. К основным таким ошибкам можно отнести:

- неперпендикулярность характерного размера тела обтекания и оси трубопровода;

- смещение оси тела обтекания преобразователя расхода относительно оси симметрии поперечного сечения трубопровода $[7,8]$.

Проанализируем погрешности, вызываемые указанными выше ошибками монтажа.

Рассмотрим ТО, изображенное на рисунке 2а. Если при проектировании ТО угол $\alpha$ выбран таким, чтобы во всем диапазоне скоростей потока срыв вихревой формации с поверхности ТО происходил на острых гранях $\mathrm{A}-\mathrm{A}$, то характерный размер ТО, определяющий частоту вихреобразования, равен $d$. В случае если плоскость А-А не перпендикулярна оси трубопровода и вектору скорости жидкости $V$, как показано на рисунке 26 , то размер $d$ изменится до значения $d^{\prime}$.

При этом относительное изменение характерного размера тела обтекания преобразователя $\Delta 1 \%$ будет равно

$$
\Delta 1 \%=\frac{d-d^{\prime}}{d} 100 \%=(1-\cos \beta) \cdot 100 \%,
$$

где $\beta$ - угол между осью трубопровода и нормалью к плоскости $\mathrm{A}-\mathrm{A}$, характеризующий неперпендикулярность характерного размера ТО и оси трубопровода, град.

Смещение оси тела обтекания преобразователя расхода относительно оси симметрии поперечного сечения трубопровода $t$ вызывает изменение рабочей площади поперечного сечения трубопровода $S_{p a \sigma}$, под которой будем понимать площадь трубопровода за вычетом площади продольного сечения тела обтекания в самой широкой его части

$$
S_{p a \tilde{\sigma}}=S-S_{T O},
$$

где $S$ - площадь сечения трубопровода, определенная измерениями или расчетом, м²; $S_{T O}$ - поперечная площадь тела обтекания по плоскости, проходящей через его наиболее широкую часть (для тела обтекания (рис. 2) поперечная площадь берется по плоскости проходящей через грани А-А), м².

Для оценки величины погрешности, возникающей вследствие смещения ТО, был проведен численный расчет для трубопроводов с условным проходом 80, 100, 150 и 200 мм и смещения оси ТО на $t$ от 1 до 5 мм относительно оси симметрии сечения трубы. Рабочая площадь трубопровода складывается из площади двух сегментов (рис. 3), с центральными углами $\alpha_{1}$ и $\alpha_{2}$, площадь которых $S_{1}$ и $S_{2}$ вычисляется по методике, изложенной в [9]. 


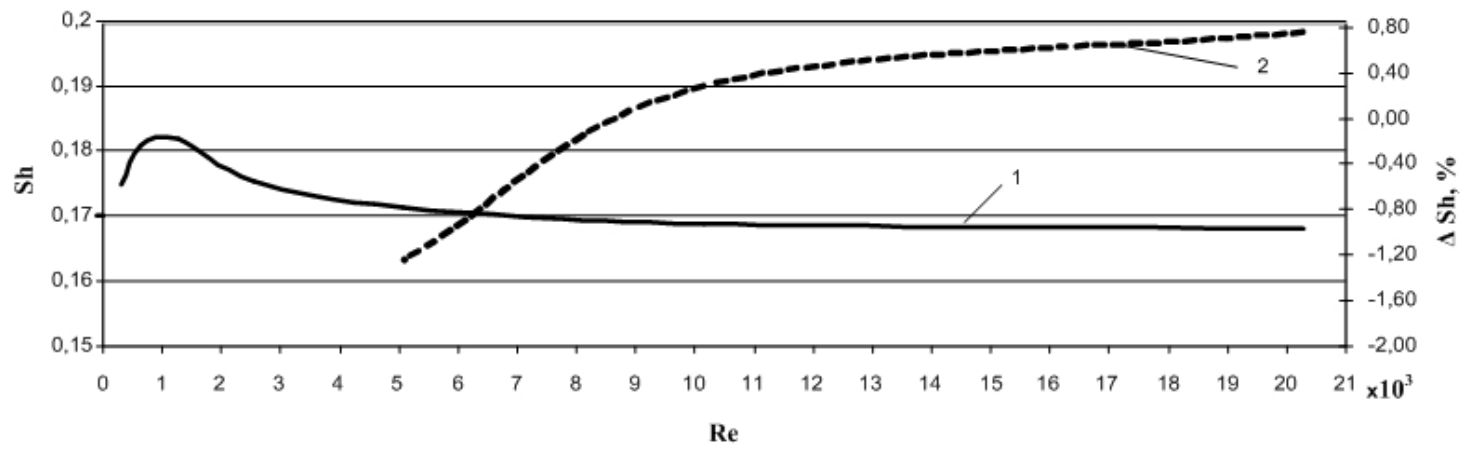

Рис. 1. График зависимости $1-S h=f(\mathrm{Re}) ; 2-\Delta S h, \%=f(\mathrm{Re})$
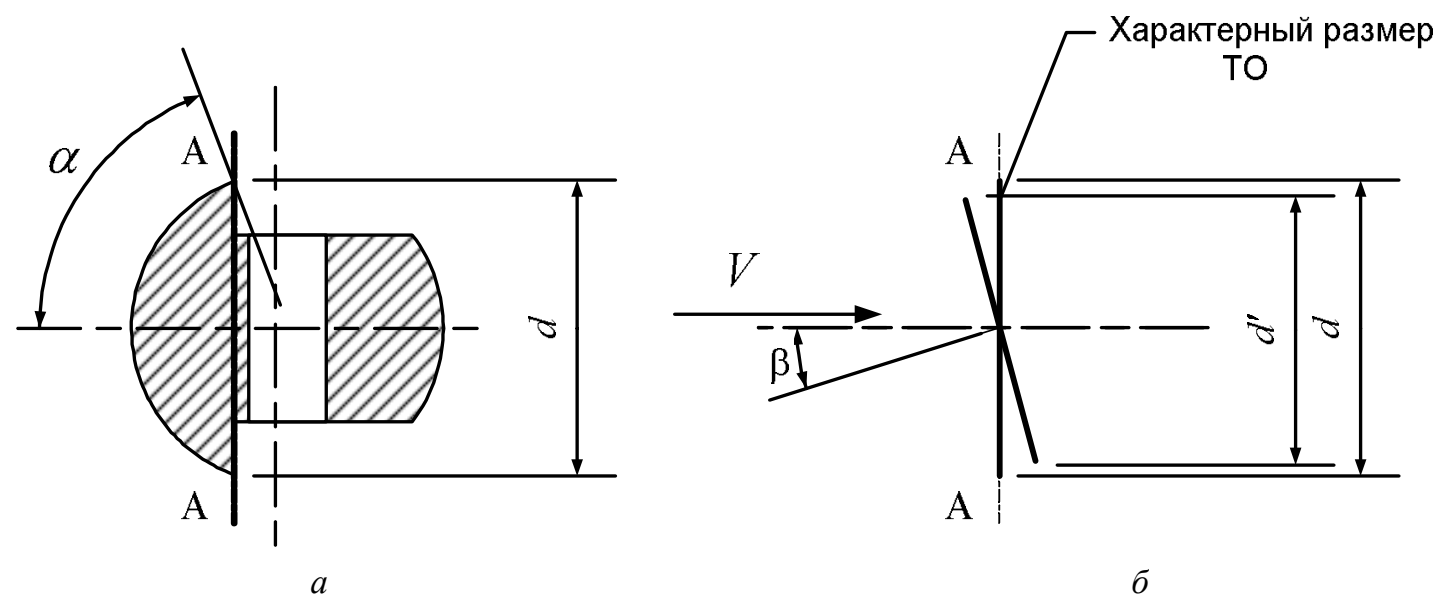

Рис. 2. Сечение тела обтекания

Относительная погрешность, возникающая в результате смещения оси ТО относительно оси трубопровода, будет такова:

$$
\Delta 2 \%=\frac{S_{p a \bar{\sigma}}(t)-S_{p a \hat{\sigma}}(0)}{S_{p a \bar{\sigma}}(0)} 100 \%,
$$

где $S_{p a \sigma}(t)$ - рабочая площадь трубопровода при смещении тело обтекания $t, \mathrm{~m}^{2} ; S_{p a \sigma}(0)$ - рабочая площадь трубопровода при нулевом смещении, м $^{2}$.

На рисунке 4 приведены результаты расчета данных погрешностей.

Рис. 3. К расчету погрешности от смещения оси ТО относительно оси симметрии поперечного сечения трубопровода






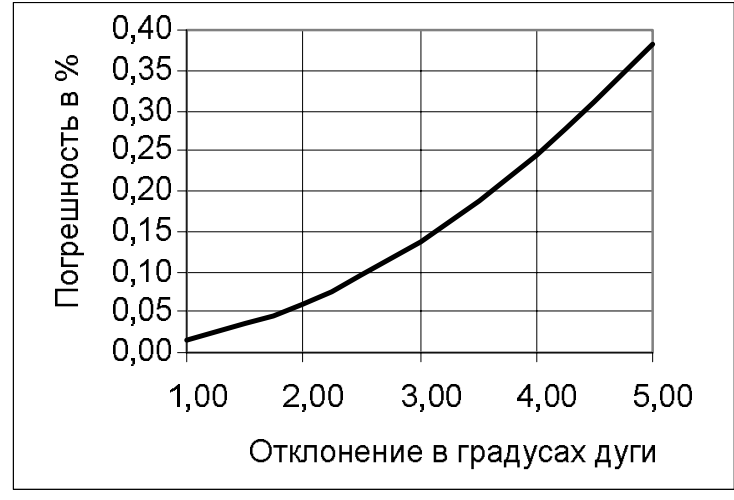

$a$

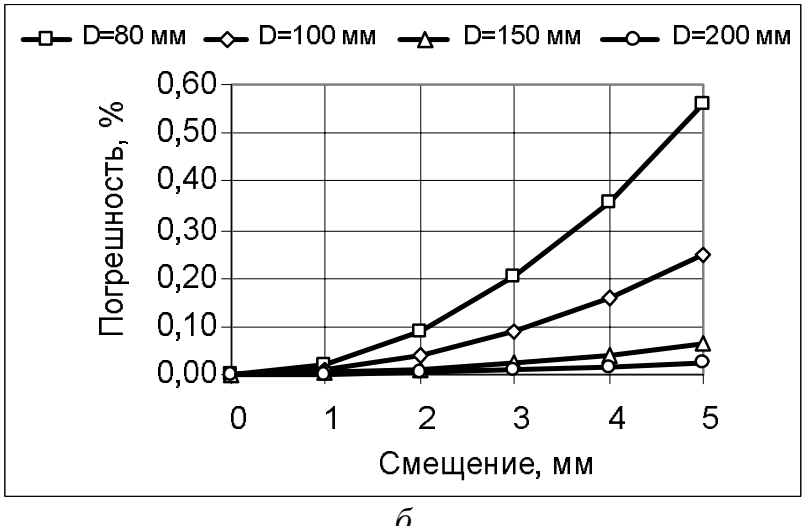

б

Рис. 4. Геометрические погрешности монтажа: $a$ - от неперпендикулярности размера $d$ и оси трубопровода; $\sigma$ - от смещения осей тела обтекания и сечения трубопровода

Рассмотренные погрешности, вызванные смещением или поворотом ТО, обусловлены изменением геометрии измерительного участка (геометрическая составляющая), поэтому могут быть точно рассчитаны. Но изменение геометрии проточной части погружного расходомера приводит и к изменению процесса вихреобразования. Возникает дополнительная погрешность, которую можно назвать гидродинамической составляющей общей погрешности. Она не может быть учтена простым расчетным путем. Определение гидродинамической составляющей погрешности может осуществляться: на проливных установках или методами численного моделирования.

Проливные установки труднодоступны, и работа на них требует больших затрат времени и средств. Поэтому в нашем исследовании мы использовали метод численного моделирования гидродинамических процессов с помощью программы Comsol Multiphysics [10]. Данное программное обеспечение позволяет моделировать самые различные задачи гидродинамики, в том числе задачи о ламинарном и турбулентном течении различных жидкостей. Например, при моделировании течений в зависимости от чисел Рейнольдса в модели может исследоваться как режим ламинарного течения (Laminar) для ньютоновских и неньютоновских жидкостей, так и различные турбулентные режимы (Turbulent). Поддерживаются несколько моделей турбулентного течения: $\mathrm{k}-\varepsilon, \mathrm{k}-\omega$ и низкорейнольдсовая low $\mathrm{k}-\varepsilon$.

При выборе моделей для расчетов мы руководствовались тем, что в оборотных водах концентрация волокна (в зависимости от стадии производства) является относительно низкой (до 1\%). В то же время в работе Ю.Д. Алашкевича и других авторов [11] показано, что в диспергированном потоке волокнистая суспензия при относительно небольших концентрациях (0,5-1\%) может рассматриваться как ньютоновская жидкость, а наличие волокна в жидкости учитывается вводом соответствующего значения вязкости жидкости.

В нашем исследовании на первом этапе использовался встроенный в программу стационарный решатель (Stationary), а на втором этапе - решатель, позволяющий исследовать временные процессы (TimeDependent). При этом в зависимости от чисел Re использовался ламинарный (Laminar) или низкорейнольдсовый (Turbulent, low $\mathrm{k}-\varepsilon$ ) режим расчета моделей.

\section{Экспериментальная часть}

Предварительные исследования показали, что большое влияние на результат моделирования оказывает эпюра скоростей жидкости на входе в измерительный участок расходомера. Поэтому мы проводили исследования в несколько этапов.

На первом этапе исследования для каждой скорости $V$, соответствующей определенному числу Re в исследуемом диапазоне, был сформирован установившийся поток жидкости и получены эпюры скоростей потока жидкости на прямом участке трубопровода длиной $100 D(D$ - диаметр трубопровода).

На втором этапе исследования моделировался процесс вихреобразования на измерительном участке расходомера. Длина измерительного участка принималась равной $6 D$, посередине которого устанавливалось ТО. Сформированная эпюра скоростей на первом этапе исследования передавалась на вход измерительного участка и проводился дальнейший расчет. 
Результатами постпроцессорной обработки данных численного эксперимента являлись:

- общая составляющая погрешности от неперпендикулярности характерного размера тело обтекания и оси трубопровода диаметром 80 и 150 мм;

- гидродинамическая погрешность от смещения осей тела обтекания и сечения трубопровода диаметром 80 мм.

В исследуемой модели рабочей жидкостью являлась волокнистая суспензия концентрацией $1 \%$ с характеристиками, описанными в работе [12].

На рисунках 5 представлены результаты численного эксперимента в виде графиков погрешности.



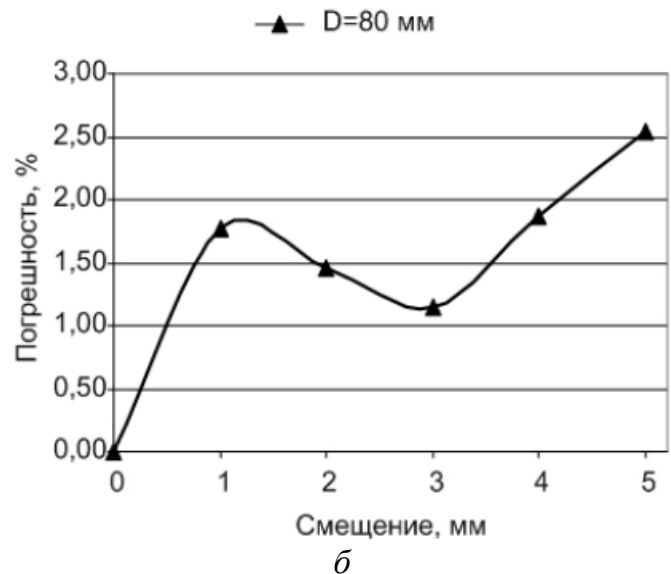

$\sigma$

Рис. 5. Общая погрешность: $a$ - от неперпендикулярности размера $d$ и оси трубопровода для $D=80$ и 150 мм; $\sigma$ - от смещения осей тела обтекания и сечения трубопровода

\section{Обсуждение результатов}

Анализируя поворот ТО относительно оси трубопровода, имеем:

- при малых углах поворота ТО (до 2 градусов, рис. 5а) погрешность $\Delta S h$ монотонно возрастает, что обусловливается неравномерностью срыва вихрей с острых граней ТО. При дальнейшем увеличении угла поворота наблюдается понижения погрешности, что является следствием ограничения вихревой формации стенками трубопровода;

- для трубопровода большого диаметра (рис. 5а) зависимость погрешности $\Delta S h$ от угла поворота ТO аналогична. Но величина самой погрешности на порядок меньше (по сравнению с $D=80$ мм), что объясняется значительно меньшим влиянием ограничения стенок трубопровода на процесс вихреобразования.

При смещении ТО относительно оси трубопровода из рисунка 5,б следует, что наблюдается резкое увеличение погрешности $\Delta S h$ (в диапазоне смещений до 1 мм), а затем ее монотонное снижение при дальнейшем увеличении смещения до 3 мм, что объясняется несимметричным ограничением процесса вихреобразования стенками трубопровода.

Выявлено, что на величину погрешности оказывает влияние не только поворот и смещение ТО, но и типоразмер трубопровода.

Кроме того, очевидно, что увеличение скорости потока жидкости также будет влиять на величину погрешности, так как при увлечении скорости изменяется режим обтекания и эпюра скоростей набегающего потока.

\section{Выводы}

1. Гидродинамическая погрешность, возникающая при ошибках монтажа, значительно превосходит расчетную геометрическую погрешность, которая учитывает только изменения геометрии измерительного участка расходомера. Поэтому данная погрешность обязательно должна определяться.

2. Гидродинамическая погрешность монтажа снижается с ростом диаметра трубопровода, поэтому дальнейшие исследование можно проводить на трубопроводах минимального типоразмера (для погружных вихревых расходомеров 80-100 мм). 
3. Погрешность монтажа как для поворота, так и для смещения ТО носит во всех случаях идентичный характер, что следует из вида полученных графиков, представленных на рисунке 5. Поскольку при любых отклонениях симметрии расположения ТО возникают схожие гидродинамические процессы.

4. Для определения допусков монтажа следует рассматривать измерительный участок расходомера как объект, на который действуют следующие входные факторы: смещение ТО, поворот относительно оси, диаметр трубопровода, скорость потока измеряемой жидкости. Выходной величиной объекта является погрешность $\Delta S h$, вызванная ошибками монтажа. Зависимость $\Delta S h$ от входных факторов следует находить методом планирования эксперимента, что может составить предмет дальнейшего исследования.

\section{Список литературы}

1. Личутина Т.Ф., Мискевич И.В., Бровко О.С., Гусакова М.А. Оптимизация нормирования сброса стоков предприятий ЦБП в водотоки. Екатеринбург, 2005. 212 с.

2. Лурье М.С., Лурье О.М., Фролов А.С. Погружные вихревые расходомеры для мониторинга сточных вод // Вода: химия и экология. 2014. №2(67). С. 108-113.

3. Лурье М.С., Елизарьева М.Ю. Расходомеры сточных вод для предприятий целлюлозно-бумажной промышленности // Экология и промышленность России. 2004. №12. С. 9-11.

4. Волынкин В.Н., Лурье М.С., Шейнин Э.М. Влияние шероховатости внутренней поверхности трубопровода на погрешность измерения погружных вихревых расходомеров // Измерительная техника. 2006. №2. С. $42-44$.

5. Лурье М.С. Оптимизация тел обтекания вихревых расходомеров для целлюлозно-бумажного производства // Химия растительного сырья. 2010. №4. С. 173-176.

6. Лурье М.С., Лурье О.М. Погрешности погружных вихревых расходомеров и методы их снижения // Датчики и системы. 2012. №1. С. 25-29.

7. Расчет и конструирование расходомеров. Л., 1977. $554 \mathrm{c.}$

8. Качество измерений: метрологическая справочная книга. Л., 1987. 295 с.

9. Бронштейн Н.И., Семендяев К.А. Справочник по математике для инженеров и учащихся втузов. М., 1980. 976 с.

10. Шмелев B.E. Femlab 2.3. Руководство пользователя. М., 1999. 442 с.

11. Алашкевич Ю.Д., Решетова Н.С., Невзоров А.И., Барановский В.П. Гидродинамические явления при безножевой обработке волокнистых материалов. Красноярск, 2004. 80 с.

12. Ерофеева А.А., Решетова Н.С., Ковалев В.И., Алашкевич Ю.Д. Определение коэффициента динамической вязкости волокнистых суспензий // Химия растительного сырья. 2010. №4. С. 177-182.

Поступило в редакиию 5 февраля 2017 г.

После переработки 11 марта 2017 2. 


\section{Alashkevich Iu.D., Lur'e M.S., Lur'e O.M., Frolov A.S. ${ }^{*}$ RELIABILITY OF MONITORING THE CIRCULATING WATERS OF PULP AND PAPER PRODUCTION WITH GRAVITY VORTEX FLOWMETERS}

M.F. Reshetnev Siberian State Aerospace University, Mira ave., 82, Krasnoyarsk, 660049 (Russia)

The paper covers the operation of submersible vortex flowmeters intended for monitoring the circulating waters of pulp and paper production.

Various components (geometric and hydrodynamic) of the overall measurement error of these flowmeters are considered. Inaccuracies in the installation of instruments as the most important operations preceding their commissioning are considered in detail. The graphs show the results of calculations of the geometric component of the error caused by the nonperpendicularity of the characteristic size of the flow body (TO) and the pipeline axis, as well as the displacement of the TO axes and the pipeline section. It is shown that a change in the geometry of the flow part of the submerged flowmeter leads to a change in the vortex formation process, which results in an additional error, which is the hydrodynamic component of the total error. To find the hydrodynamic component of the error, the method of numerical simulation of hydrodynamic processes was used. Dependencies of the installation error on the hydrodynamic component of the total error are presented. It is revealed that the error value is influenced not only by rotation and displacement of the TO, but also by the size of the pipeline, which in turn is related to the process of vortex formation.

Keywords: circulating water, flow body, vortex flowmeters, installation errors, simulation simulation.

\section{References}

1. Lichutina T.F., Miskevich I.V., Brovko O.S., Gusakova M.A. Optimizatsiia normirovaniia sbrosa stokov predpriiatii $T s B P v$ vodotoki. [Optimization of the normalization of discharge of sewage from pulp and paper enterprises into watercourses]. Ekaterinburg, 2005, 212 p. (in Russ).

2. Lur'e M.S., Lur'e O.M., Frolov A.S. Voda: khimiia i ekologiia, 2014, no. 2(67), pp. 108-113. (in Russ).

3. Lur'e M.S., Elizar'eva M.Iu. Ekologiia i promyshlennost' Rossii, 2004, no. 12, pp. 9-11. (in Russ).

4. Volynkin V.N., Lur'e M.S., Sheinin E.M. Izmeritel'naia tekhnika, 2006, no. 2, pp. 42-44. (in Russ).

5. Lur'e M.S. Khimiia rastitel'nogo syr'ia, 2010, no. 4, pp. 173-176. (in Russ).

6. Lur'e M.S., Lur'e O.M. Datchiki i sistemy, 2012, no. 1, pp. 25-29. (in Russ).

7. Raschet i konstruirovanie raskhodomerov. [Calculation and design of flowmeters]. Leningrad, 1977, 554 p. (in Russ).

8. Kachestvo izmerenii. (Metrologicheskaia spravochnaia kniga). [The quality of measurements. (Metrological reference book)]. Leningrad, 1987, 295 p. (in Russ).

9. Bronshtein N.I., Semendiaev K.A. Spravochnik po matematike dlia inzhenerov $i$ uchashchikhsia vtuzov. [A handbook on mathematics for engineers and students of technical colleges]. Moscow, 1980, 976 p. (in Russ).

10. Shmelev V.E. Femlab 2.3. Rukovodstvo pol'zovatelia. [Femlab 2.3. User guide]. Moscow, 1999, 442 p. (in Russ).

11. Alashkevich Iu.D., Reshetova N.S., Nevzorov A.I., Baranovskii V.P. Gidrodinamicheskie iavleniia pri beznozhevoi obrabotke voloknistykh materialov. [Hydrodynamic phenomena in knifeless processing of fibrous materials]. Krasnoyarsk, 2004, 80 p. (in Russ).

12. Erofeeva A.A., Reshetova N.S., Kovalev V.I., Alashkevich Iu.D. Khimiia rastitel'nogo syr'ia, 2010, no. 4, pp. 177-182.

Received February 5, 2017

Revised March 11, 2017

\footnotetext{
* Corresponding author.
} 
